
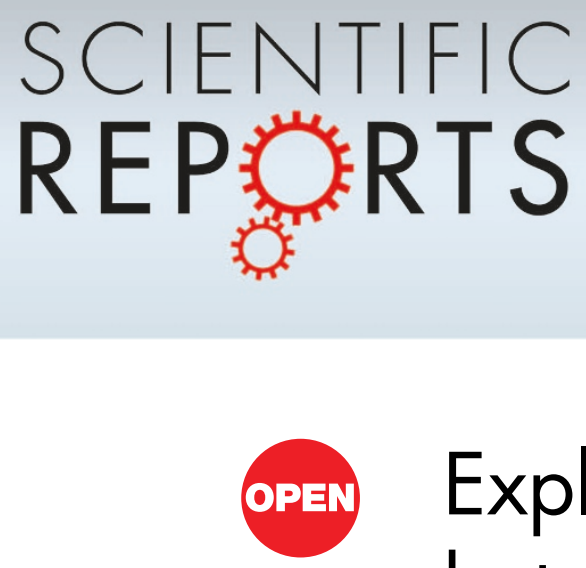

\title{
Exploring structure-function relationships between TRP and Kv channels
}

SUBJECT AREAS:

ION TRANSPORT

ION CHANNELS IN THE NERVOUS SYSTEM

NEUROPHYSIOLOGY

MOLECULAR NEUROSCIENCE

Received

5 February 2013

Accepted

7 March 2013

Published

22 March 2013

Correspondence and requests for materials should be addressed to K.J.S. (swartzk@ninds. nih.gov)

\author{
Jeet Kalia \& Kenton J. Swartz
}

Porter Neuroscience Research Center, Molecular Physiology and Biophysics Section, National Institute of Neurological Disorders and Stroke, National Institutes of Health 35 Convent Drive, Bethesda, Maryland 20892, USA.

The molecular mechanisms underlying the activation of Transient Receptor Potential (TRP) ion channels are poorly understood when compared to those of the voltage-activated potassium $(\mathrm{Kv})$ channels. The architectural and pharmacological similarities between the members of these two families of channels suggest that their structure-function relationships may have common features. We explored this hypothesis by replacing previously identified domains and critical structural motifs of the membrane-spanning portions of Kv2.1 with corresponding regions of two TRP channels, TRPM8 and TRPV1. Our results show that the S3b-S4 paddle motif of Kv2.1, but not other domains, can be replaced by the analogous regions of both TRP channels without abolishing voltage-activation. In contrast, replacement of portions of TRP channels with those of Kv2.1 consistently yielded non-functional channels. Taken together, these results suggest that most structural elements within TRP channels and Kv channels are not sufficiently related to allow for the creation of hybrid channels.

T ransient Receptor Potential (TRP) channels have been the intense focus of research since the first member was cloned in $1989^{1}$. Despite these efforts, the structural and mechanistic basis of TRP channel function remains poorly understood, in part because we currently have limited high resolution structural information on these channels ${ }^{2}$. In addition, TRP channels are modulated by a vast array of ligands possessing disparate physical and chemical characteristics, making it difficult to localize their binding sites and establish their mechanisms of activation. For example, TRPV1 is activated by stimuli as diverse as voltage, heat, protons, vanilloid compounds such as capsaicin and resiniferatoxin (RTX), and peptide toxins such as the double-knot toxin (DkTx) and vanillotoxins ${ }^{3-6}$. Another thermosensitive TRP channel, TRPM8, is activated by voltage, cold, and the small organic compounds, menthol and icilin ${ }^{7}$. How such a vast array of stimuli can activate these channels remains fascinating and poorly understood. Mutagenesis- and chimera-based approaches have identified regions of these channels that play critical roles in channel activation. For example, these approaches have been used to identify residues in TRPV1 that are critical for its activation by ligands such as capsaicin ${ }^{8,9}, \mathrm{RTX}^{9,10}, \mathrm{DkTx}^{11}$, temperature ${ }^{12-14}$ and $\mathrm{pH}^{15}$. Similar studies on TRPM8 have identified channel residues that are important for its activation by voltage ${ }^{16}$ and its chemical agonists, icilin $^{17}$ and menthol ${ }^{18}$. Although this information is extremely valuable, it remains a challenge to discern whether the residues identified are directly involved in ligand binding or whether they influence an allosteric transition involved in channel gating. This task is especially non-trivial in the context of TRP channels because the gating elements in these channels remain largely unidentified.

In attempting to understand the principles underlying the activation of TRP channels, we sought to draw on our knowledge of voltage-activated potassium $(\mathrm{Kv})$ channels, a family of ion channels that have been subject to extensive biophysical and structural investigation ${ }^{19}$. Several lines of evidence suggest that $\mathrm{Kv}$ channels and TRP channels may exhibit structural and functional similarities. First, members of both these channel families possess tetrameric architectures where each monomer consists of six transmembrane segments (S1-S6) with the S5-S6

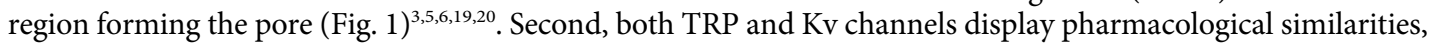
such as modulation by isostructural cystine knot peptide toxins- $\mathrm{Kv}$ channels are inhibited by voltage sensorbinding toxins ${ }^{19,21}$, and TRPV1 is activated by double-knot toxin $(\mathrm{DkTx})^{11,22}$ and vanillotoxin ${ }^{23}$. Indeed, individual vanillotoxins have been reported to cross-react with TRPV1 and Kv2.1 ${ }^{23}$. Another TRP channel, TRPA1, is activated by the tarantula toxin, GsMtx- $4^{24}$. An additional pharmacological similarity between TRP and $\mathrm{Kv}$ channels is that members of both families are inhibited by internal quaternary ammonium ions ${ }^{25-27}$. A third line of evidence supporting similarities between TRP channels and $\mathrm{Kv}$ channels is that residues important for ligandmodulation of these channels map to similar regions. For example, residues in the S3-S4 region of TRPV1 (Fig. 2, green residues) play important roles in its activation by capsaicin and $\mathrm{RTX}^{9,10}$, and several S3 and S4 residues of TRPM8 are important for menthol and icilin-sensitivity of the channel ${ }^{16,18}$ (Fig. 2, orange and blue residues, 


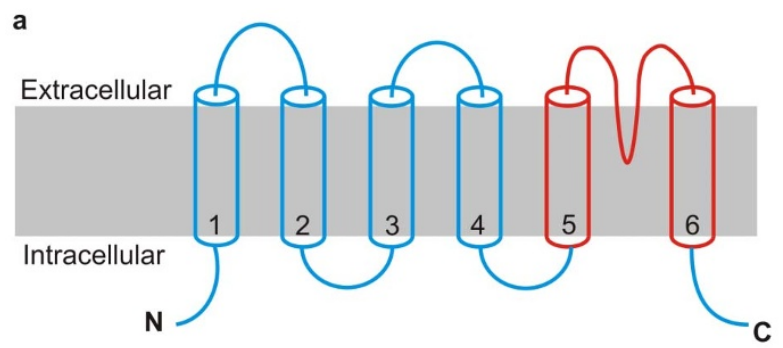

b

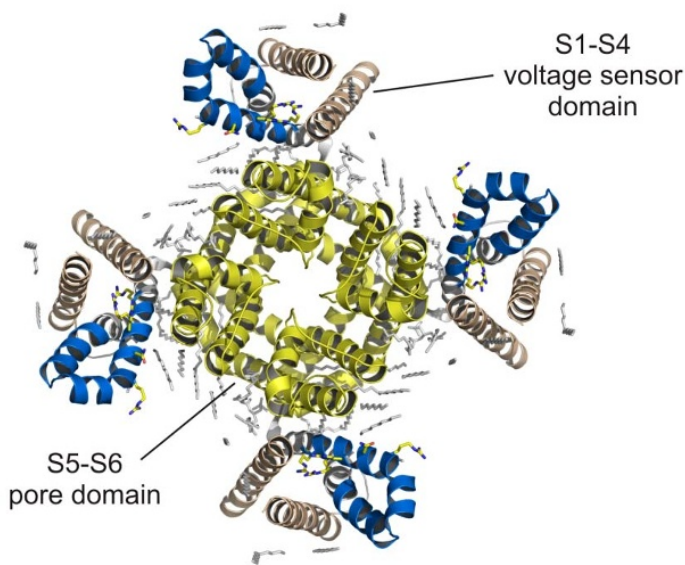

Figure $1 \mid$ Architecture of Kv and TRP channels. (a) Schematic representation of the transmembrane topology of channel subunits containing six transmembrane segments with the pore region formed by S5 and S6 segments shown in red. (b) Crystal structure of the tetrameric assembly of the Kv1.2-Kv2.1 chimera (Accession code 2R9R) ${ }^{20}$ viewed from the extracellular side. The S3b-S4 paddle motif is shown in blue.

respectively). These regions overlap with the receptor for voltage sensor-targeting toxins in $\mathrm{Kv}$ channels-the S3b-S4 "paddle" (Fig. 1b; Fig. 2, gray highlighted region), a helix-turn-helix motif that moves in response to changes in membrane voltage to drive opening of the channel ${ }^{19,28-35}$. Fourth, charge-neutralizing mutations of positively charged residues in the S4 helix of TRPM8 have been shown to reduce the amount of charge that moves during voltage-activation of the channel ${ }^{16}$, suggesting that this TRP channel's S4 helix may function as a voltage sensor, similar to what has been established in $\mathrm{Kv}$ channels ${ }^{19,36-38}$. Finally, studies on TRPV $1^{26,39}$ demonstrate that the internal pore is formed by $\mathrm{S} 6$ and that it opens and closes in response to capsaicin or voltage, paralleling the evidence for the S6 region of $\mathrm{Kv}$ channels forming the pore and serving as a gate that limits the flow of ions in the closed states ${ }^{40}$. Collectively, these intriguing observations support the idea that the transmembrane regions of TRP channels and $\mathrm{Kv}$ channels may have similar structures and that their mechanisms of gating may be related.

In the present study we explored the relationships between TRP channels and $\mathrm{Kv}$ channels using a chimera approach to determine whether structural motifs can be transferred between the two families of cation channels without disrupting function. Our efforts were motivated by previous chimera studies on a range of distantly related voltage-activated ion channels and voltage sensing proteins that have provided valuable information on structural relationships and in defining domains or motifs that serve specific functions ${ }^{32,34,41,42}$.

\section{Results}

We decided to focus our efforts on the Kv2.1 channel and on two TRP channels, TRPV1 and TRPM8. We chose Kv2.1 because the gating properties of this $\mathrm{Kv}$ channel can be modulated by an array of peptide toxins that interact with the S1-S4 voltage-sensing domain $^{21}$, and because earlier studies have successfully used this channel to generate chimeras with other voltage-activated cation channels and voltage-sensitive proteins ${ }^{32,34}$. Our choice of TRPV1 was motivated by the availability of a large number of pharmacological tools targeting this channel, including vanilloid compounds and $\mathrm{DkTx}^{4,6,11,22}$. TRPM8 was an obvious choice for our studies because an earlier report suggested similar voltage-sensing mechanisms in this channel and Kv channels ${ }^{16}$.

Fig. 2 shows the primary sequence alignment used to generate chimeras, covering the S1-S6 transmembrane segments of TRPV1, TRPM8 and Kv2.1, along with a variety of other tetrameric cation channels and voltage-sensitive proteins, including those for which Xray structures are available ${ }^{20,28,43-45}$. Fig. 3 summarizes all 50 chimeras that we generated, and provides the specific boundaries for regions within the S1-S6 segments that were transferred between Kv2.1 and either TRPV1 or TRPM8. Unless otherwise stated, channel constructs were investigated by injecting cRNA into oocytes and performing two-electrode voltage clamp recordings to investigate their functional properties.

S3-S4 chimeras between TRPM8 and Kv2.1. The S3b-S4 paddle motif in $\mathrm{Kv}$ channels is extremely tolerant to protein engineering because it is relatively structurally unconstrained, making few contacts with other parts of the protein ${ }^{20,28,29,32,34,43}$. Indeed, in earlier work, the paddle region of Kv2.1 was replaced by the paddle regions of other voltage-gated channels such as the prokaryotic $\mathrm{Kv}$ channel, KvAP, Nav channels, the voltage-activated proton channel Hv1, and the voltage-sensitive phosphatase Ci-VSP, without destruction of voltage-activation and with concomitant transfer of pharmacology ${ }^{32,34}$. Due to its functional and pharmacological importance, and its tolerance to replacement, we first focused on making chimeras by replacing the paddle region of Kv2.1 with the corresponding regions of TRPV1 and TRPM8.

We generated twelve chimeras in which different portions of the Kv2.1 paddle were replaced with portions of the putative S3-S4 region of TRPM8 (Fig. 3d; 1-12M8Kv). Seven of these chimeras gave rise to functional channels (Fig. $3 \mathrm{~d}$; green dots) that were activated by membrane depolarization and that were sensitive to the selective $\mathrm{Kv}$ channel blocker, agitoxin $2^{46}$. The voltage-activated currents observed for these chimeras exhibited a reversal potential of $\sim-20 \mathrm{mV}$, consistent with the expected value for $\mathrm{K}^{+}$- selective channels for the recording solution we used. All of these functional chimeras involved replacing regions within and immediately $\mathrm{N}$-terminal to the paddle region $(2 \mathrm{M} 8 \mathrm{Kv}, 3 \mathrm{M} 8 \mathrm{Kv}, 5 \mathrm{M} 8 \mathrm{Kv}, 7 \mathrm{M} 8 \mathrm{Kv}, 8 \mathrm{M} 8 \mathrm{Kv}, 11 \mathrm{M} 8 \mathrm{Kv}$, and $12 \mathrm{M} 8 \mathrm{Kv}$ ), whereas those that failed to form functional channels $(1 \mathrm{M} 8 \mathrm{Kv}, 4 \mathrm{M} 8 \mathrm{Kv}, 6 \mathrm{M} 8 \mathrm{Kv}, 9 \mathrm{M} 8 \mathrm{Kv}$, and $10 \mathrm{M} 8 \mathrm{Kv})$ involved the transfer of regions extending beyond previously defined boundaries of the paddle motif ${ }^{32}$.

Two of the largest functional paddle chimeras were $8 \mathrm{M} 8 \mathrm{Kv}$, a construct in which 31 residues of the paddle were replaced by 34 residues of TRPM8, and $3 \mathrm{M} 8 \mathrm{Kv}$, a construct in which 37 residues of Kv2.1 were replaced by 40 residues of TRPM8 (Fig. 4a). Although both of these chimeras were activated by membrane depolarization, their gating characteristics were different from those of Kv2.1. Indeed, both the chimeras exhibited much slower rates of activation and deactivation (Fig. 4b, c, and d; note differences in scale bars) and their conductance-voltage $(\mathrm{G}-\mathrm{V})$ relations had much shallower slopes compared to that of Kv2.1 (Fig. 4e). Fitting of a Boltzmann function to the $\mathrm{G}-\mathrm{V}$ data for $3 \mathrm{M} 8 \mathrm{Kv}$ and $8 \mathrm{M} 8 \mathrm{Kv}$ yielded slopes $(z)$ of 1.5 and 1.7, respectively, as compared to 3.2 for Kv2.1 (Fig. 4e; Table 1). One possible explanation for the reduced $z$ values of these chimeras is that the three outer arginine residues in the S4 helix of Kv2.1 were replaced by only one positively charged residue in the transplanted region of TRPM8 (Fig. 4a). The energetics of gating were also perturbed in these chimeras; whereas $8 \mathrm{M} 8 \mathrm{Kv}$ could be activated by a voltage stimulus lower than that required to activate 
(177) KPNSSVAAKILAIISIMFIVLSTIALSL---------NTLPELQSLDEFGQSTDN-------PQLAHVEAVCIAWFTMEYLLRFLSS----

(155) YPESSGPARI IAIVSVMVILISIVSFCLETLPIFRDENEDMHGGGVTFHTYSNSTIGYQQSTSFTDPFFIVETLCI IWFSFEFLVRFFAC----

(219) YPESSQAARVVAI ISVFVILLSIVIFCLETLPEFKHYKVFNTTTNGT-------KIEEDEVPDITDPFFLIETLCI IWETFELTVRFLAC----

(30) NIGDVMEHPLVELGVSYAALLSVIVVVVEYTMQLSGEYL-_-n-

(108) RVRAVIDHLGMRVFGVFLIFLDI ILMIIDLSLPGKSESSQ----------------------SFYDGMALALSCYFMLDLGLRIFAY----

(91) MLRKLFSSHRFQVI I ICLVVLDALLVLAEL I LDLKI IQPDKN----------------NYAAMVFHYMS IT ILVFFMMEI IFKLFVF----

(121) LAIKILVHSLFNVLIMCTILTNCVFMTMSNPPDWTK------------------------NVEYTFTGIYTFESLIKILARGFCLE---

(27) VESS---FFTKFI IYLIVLNGITMGLETSKTFMQSFG-----------------------VYTTLFNQIVITIFTIEIILRIYV----

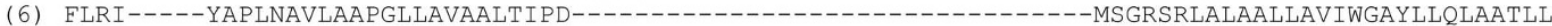

NavAb

Mlotik

TRPV1

TRPM8

Kv2.1

Kv1.2

Shaker

\section{monsinn-}

\begin{tabular}{|c|c|}
\hline (498) & VLYF----SQRKEYVASMVFSLAM---------GWTNMLYYTRGFQQMGIYAVMIEKMI------- \\
\hline$(785)$ & HSVPHTVNYFT-DLWNVMDTLGLFYFIAGIVFRLHS--SNKSSLYSGRVIF-----------CLDYIIFTLRIIHIFTVSRNLGPKI IMLQRM-- \\
\hline 48) & PKKWKFFK----GPLNAIDLLAILPYYVTIFLTE----SNKSVLQFQNVR-------------RVVQIFRIMRILRILKLARHSTGLQSLGFTLRR \\
\hline 45) & PSKAGFFT----NIMNIIDIVAI IPYFITLGTELAE--KPEDAQQGQQAMSL-----------AILRVIRLVRVFRIFKLSRHSKGLQILGQTLKA \\
\hline 02$)$ & PNKLNFCR----DVMNVIDI IAI I PYFITLATVVAE--EEDTLNLPKAPVSPQDKSSNQAMSLA ILRVIRLVRVFRI FKLSRHSKGLQILGRTLKA \\
\hline (95) & DPAGYVK------KTLYEIPALVPAGLLALIEGHLAG--LG------------------LFRLVRLLRFLRILLI ISRGSKFLSAIADAA- \\
\hline (173) & GPKNFFT-----NPWEVADGLI IVVTFVVTIFYTVLDEYVQETGADGLG-------------RLVVLARLLRVVRLARIFYSHQQMKASSRRTIS \\
\hline 82$)$ & DFTELRNNPWNWLDFTVITFAYVTEFV-----------NLGNVS---------------------ALRTFRVLRALKTISVIPGLKTIVGALIQ \\
\hline 85) & HRISFFKDPWSLFDFFVVAISLV--------------PTSSGF--------------------EILRVLRVLRLFRLVTAVPQMRKIVSALIS \\
\hline 62) & 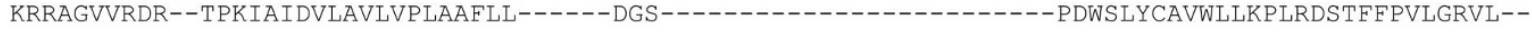 \\
\hline
\end{tabular}

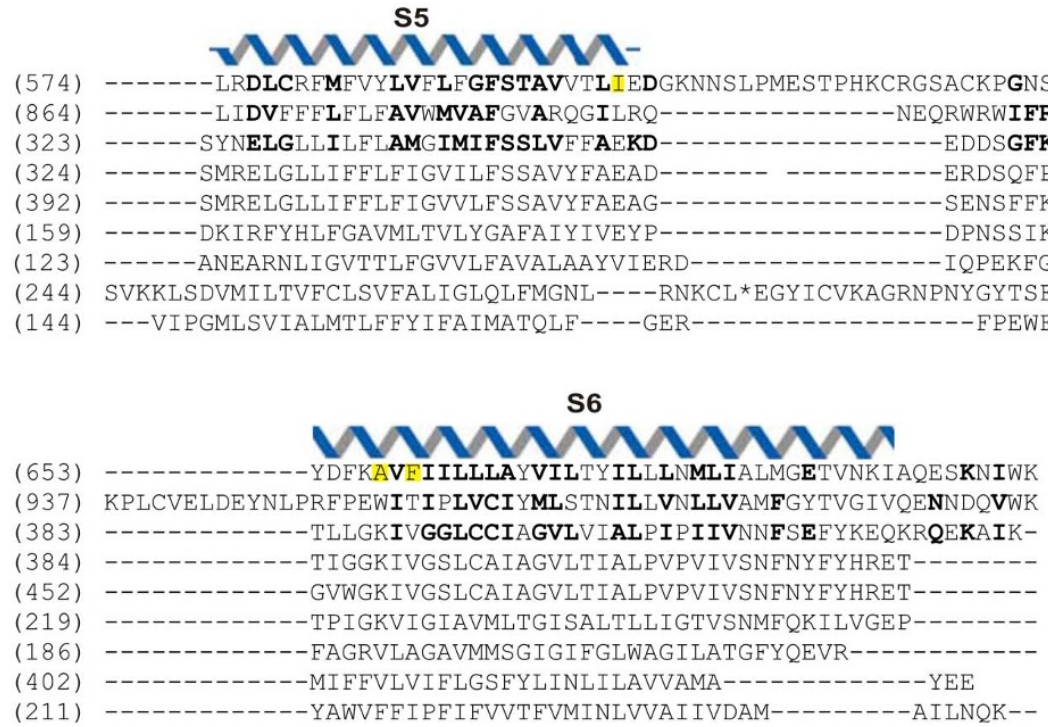

TRPV1

*: QWPPDNSTFEINITSFFNNSLDWNGTAFNRTVNMFNWDEYIEDKSHFYFLEGQNDALLCGNSSDAGQCP

Figure $2 \mid$ Sequence alignment of six transmembrane-tetrameric ion channels and voltage-sensitive proteins. Residues of TRPV1 and TRPM8 that are identical or similar to those of Kv2.1 to which they are aligned are shown in bold lettering. The residues belonging to the selectivity filter region of each channel are underlined. Conserved residues that are important for voltage sensing in voltage-activated ion channels are depicted in red. TRPV1 residues shown in green are important for vanilloid sensitivity and those highlighted in yellow background are critical for DkTx sensitivity. TRPM8 residues shown in blue and orange are important for icilin and menthol sensitivity, respectively. A portion of the unusually long linker between the $\mathrm{S} 5$ and S6 helices of rNav1.2 (marked by an asterisk) is shown separately at the bottom for clarity.

Kv2.1, 3M8Kv required stronger depolarizations to elicit voltageactivated currents (Fig. 4e; Table 1). The large rightward shift of the $\mathrm{G}-\mathrm{V}$ relationship of $3 \mathrm{M} 8 \mathrm{Kv}$ precludes utilization of agitoxin2 to subtract background currents because the toxin unbinds at the higher voltages required to activate this chimera. Interestingly, this chimera remains constitutively open and cannot be closed entirely by membrane hyperpolarization, giving rise to a steady holding current (Fig. $4 \mathrm{~d}$ and $\mathrm{f}$ ) and non-zero conductance values at negative voltages (G-V plot in Fig. 4e). To verify that this holding current arises from the chimera, we applied agitoxin2 and observed that the holding current was reduced to negligible values (not shown).
In addition to playing important roles in sensing voltage, the S4 helix of TRPM8 is thought to be important for menthol sensitivity ${ }^{16}$, raising the possibility that the transferred region of TRPM8 may confer ligand sensitivity to the chimeras. We therefore examined the sensitivity of the functional chimeras to menthol and in each case external application of the TRPM8 agonist was without effect. Voltage-activated currents before and after menthol treatment for our largest paddle chimera, $3 \mathrm{M} 8 \mathrm{Kv}$, are depicted in Fig. $4 \mathrm{f}$.

If the S4 helix of TRPM8 serves as the voltage sensor of TRPM8, we might expect a TRPM8 variant containing a larger number of positively charged residues to display steeper voltage-dependent gating. 


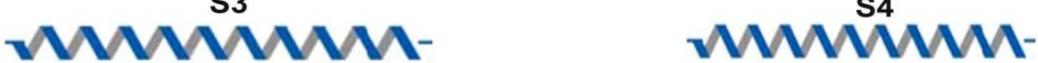

TRPV1

TRPM8

Kv2.1
(498) QRRPSLKSLFVDSYSEILFFVQSLFMLVSVVLYF--SQR

HSVPHTVN-YFTDLWNVMDTLGLFYFIAGIVFRLHSSNKSSLYSGRVIF---CLDYI I FTLRLIHIFTVSRNLGPKI IMLQRM

(248) PKKWK----FFKGPLNAIDLLAILPYYVTIFLTE--SNKSVLQFQNVR----RVVQIFRIMRILRILKLARHSTGLQSLGFTLRRS $\begin{array}{lll}7 & 8 & 10 \\ & 9 & \end{array}$

11213

$\begin{array}{llllll}15 & 16 & 20 & 24 & 26 & 28\end{array}$

$\begin{array}{llll}17 & 21 & 25 & 27\end{array}$

$18 \quad 22$

1923 b

TRPV1 Kv2.1

\section{S3}

rumpin-
MMMNMM-

munn-

(498) QRRPSLKSLFVDSYSEILFFVQSLFMLVSVVLYFSQRKEYVASM--VFSLAMGWTNMLYYTRGFQQMGIYAVMIEKMI

(248) PKKWK----FFKGPLNAIDLLAILPYYVTIFLTESNKSVLQFQNVRRVVQIFRIMRILRILKLARHSTGLQSLGFTLRRS
33

c

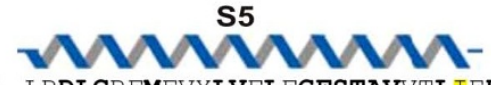

TRPV1 (574) LRDLCREMFVYLVFLFGFSTAVVTLIEDGKNNSLPMESTPHKCRGSACKPGNSYNSLYSTCLELFKFTIGMGDLEFT--

TRPM8 (864) LIDVFFFLFLFAVWMVAFGVARQGILRQ----------NEQRWRWIFRSVIYEPYLAMFGQVP-SDVDSTTYDF

KV2.1 (324) YNELGLLILFLAMGIMIFSSLVFFAEKD---------------EDDSGFKSIPDAFWWATITMTTVGYGDMT----

34

35

36

\section{S6}

MMMMMM

TRPV1 (653) ---------------ENYDFKAVFIILLLAYVILTYILLLNMLIALMGETVNKIAQESKNIWK

TRPM8 (950) SHCTFSGNESKPLCVELDEYNLPRFPEWITIPLVCIYMLSTNILLVNLLVAMFGYTVGIVQENNDQVWK

KV2.1 (383) ----------------PVTLLGKIVGGLCCIAGVLVIALPI PIIVNNFSEFYKEQKRQEKAIK-

$37 \quad 38$

39

40

d

\begin{tabular}{|c|c|c|c|c|c|c|c|}
\hline & \multicolumn{2}{|c|}{ TRPM8 in Kv 2.1} & \multicolumn{2}{|c|}{ TRPV1 in Kv 2.1} & Kv2.1 in TRPM8 & \multicolumn{2}{|c|}{ Kv2.1 in TRPV1 } \\
\hline S3-S4 chimeras & $\begin{array}{l}(6,11) \\
(7,11) \\
(7,21) \\
(7,22) \\
(9,24) \\
(9,25) \\
(10,19) \\
(10,21) \\
(10,26) \\
(20,28) \\
(13,21) \\
(14,22)\end{array}$ & $\begin{array}{r}1 \mathrm{M} 8 \mathrm{KV} \\
2 \mathrm{M} 8 \mathrm{KV} \\
3 \mathrm{M} 8 \mathrm{KV} \\
4 \mathrm{M} 8 \mathrm{KV} \\
5 \mathrm{M} 8 \mathrm{KV} \\
6 \mathrm{M} 8 \mathrm{KV} \\
7 \mathrm{M} 8 \mathrm{KV} \\
8 \mathrm{M} 8 \mathrm{KV} \\
9 \mathrm{M} 8 \mathrm{KV} \\
10 \mathrm{M} 8 \mathrm{KV} \\
11 \mathrm{M} 8 \mathrm{KV} \\
12 \mathrm{M} 8 \mathrm{KV}\end{array}$ & $\begin{array}{r}(8,21) \\
(10,21 \\
(29,32 \\
(30,33 \\
(30,32 \\
(31,32\end{array}$ & $\begin{array}{l}1 \mathrm{~V} 1 \mathrm{KV} \\
2 \mathrm{~V} 1 \mathrm{KV} \\
3 \mathrm{~V} 1 \mathrm{KV} \\
4 \mathrm{~V} 1 \mathrm{KV} \\
5 \mathrm{~V} 1 \mathrm{KV} \\
6 \mathrm{~V} 1 \mathrm{KV}\end{array}$ & $\begin{array}{ll}(10,21) & 1 \text { KvM8 } \\
(12,18) & \text { 2KvM8 } \\
(17,26) & 3 \mathrm{KvM} 8 \\
(20,26) & 4 \mathrm{KvM} 8\end{array}$ & $\begin{array}{l}(10,21) \\
(15,23) \\
(17,27) \\
(31,32)\end{array}$ & $\begin{array}{l}1 \mathrm{KvV1} \\
2 \mathrm{KvV1} \\
3 \mathrm{KvV1} \\
4 \mathrm{KvV1}\end{array}$ \\
\hline S2-S3 chimeras & - & & $\begin{array}{l}(3,4) \\
(3,5)\end{array}$ & $\begin{array}{l}7 \mathrm{~V} 1 \mathrm{KV} \\
8 \mathrm{~V} 1 \mathrm{KV}\end{array}$ & - & - & \\
\hline S1-S4 chimeras & $\begin{array}{l}(1,21) \\
(1,27)\end{array}$ & $\begin{array}{l}13 \mathrm{M} 8 \mathrm{KV} \\
14 \mathrm{M} 8 \mathrm{KV}\end{array}$ & $\begin{array}{l}(1,21) \\
(1,27)\end{array}$ & $\begin{array}{r}9 \mathrm{~V} 1 \mathrm{KV} \\
10 \mathrm{~V} 1 \mathrm{KV}\end{array}$ & $\begin{array}{ll}(1,21) & 5 \mathrm{KvM} 8 \\
(1,27) & 6 \mathrm{KvM} 8\end{array}$ & $\begin{array}{l}(1,21) \\
(1,27)\end{array}$ & $\begin{array}{l}5 \mathrm{KvV1} \\
6 \mathrm{KvV1}\end{array}$ \\
\hline S2-S4 chimeras & $(2,21)$ & $15 \mathrm{M} 8 \mathrm{KV}$ & $(2,21)$ & $11 \mathrm{~V} 1 \mathrm{KV}$ & $(2,21) \quad 7 \mathrm{KvM} 8$ & $(2,21)$ & $7 \mathrm{KvV1}$ \\
\hline S5-S6 chimeras & $\begin{array}{l}(34,40) \\
(35,39) \\
(36,37)\end{array}$ & $\begin{array}{l}16 \mathrm{M} 8 \mathrm{KV} \\
17 \mathrm{M} 8 \mathrm{KV} \\
18 \mathrm{M} 8 \mathrm{KV}\end{array}$ & $\begin{array}{l}(34,40) \\
(35,38) \\
(36,37)\end{array}$ & $\begin{array}{l}12 \mathrm{~V} 1 \mathrm{KV} \\
13 \mathrm{~V} 1 \mathrm{KV} \\
14 \mathrm{~V} 1 \mathrm{KV}\end{array}$ & 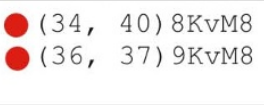 & 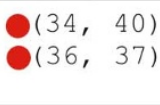 & $\begin{array}{l}8 \mathrm{KvV1} \\
9 \mathrm{KvV1}\end{array}$ \\
\hline
\end{tabular}

Figure 3 Chimeras generated and characterized in this study. (a) Alignment of S1-S4 regions of TRPV1, TRPM8 and Kv2.1 used for generating most chimeras. (b) An alternate alignment of the S3-S4 regions of TRPV1 and Kv2.1. (c) Alignment of the S5-S6 regions for TRPV1, TRPM8 and Kv2.1. The numbers below specified residues in the alignment of the channels denote the start or end sites of the swapped regions. (d) Summary of chimeras generated in this study. Red dots indicate non-functional chimeras whereas green dots indicate functional chimeras. The chimeras are named using the code: $(a, b) X C 1 C 2$, where numbers ' $a$ ' and ' $b$ ' correspond to the $\mathrm{N}$-terminal residue and the C-terminal residue respectively of the transferred segment, ' $\mathrm{X}$ ' is the serial number, ' $\mathrm{C} 1$ ' is the abbreviation for the donor protein and ' $\mathrm{C} 2$ ' is the abbreviation for the acceptor protein. Abbreviations for the proteins are: M8 for TRPM8, V1 for TRPV1 and Kv for Kv2.1. 
TRPM8 (793) DLWNVMDTIGLFYFTAGIVFRLHSSNKSSLYSGRVIFCLDYIT FTLRITHIFTVSRN

KV2. 1 (253) GPLNAIDLLAILPYYVTIFLTE--SNKSVLQFQNVR-RVVQIFRIMRILRILKLARH

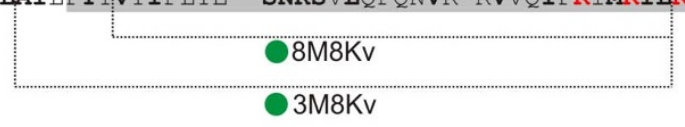

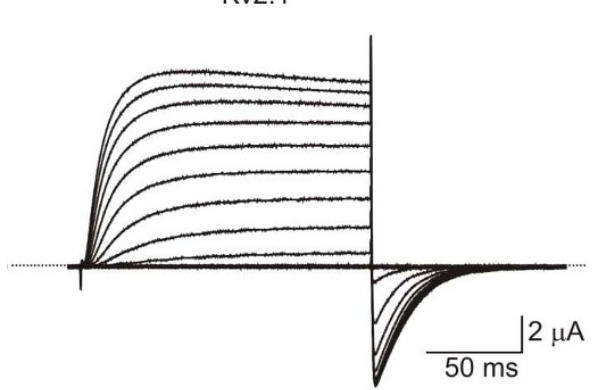

C

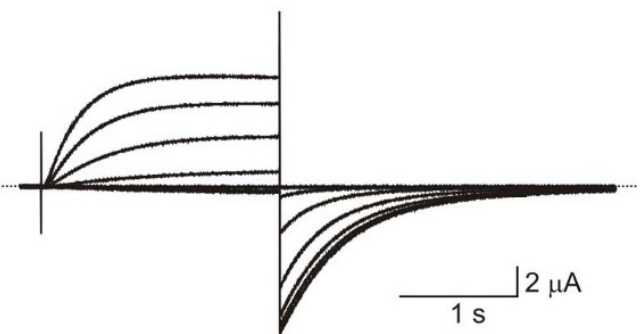

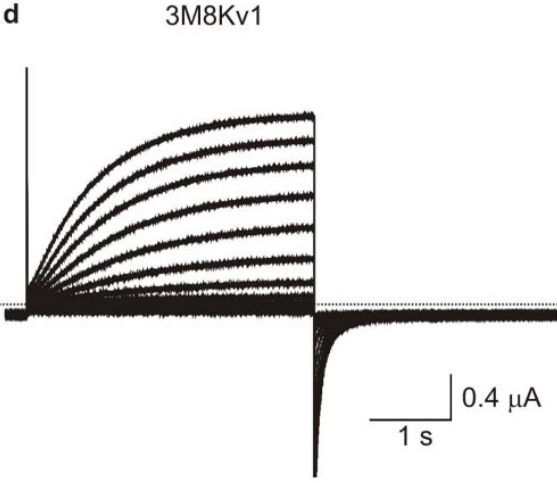

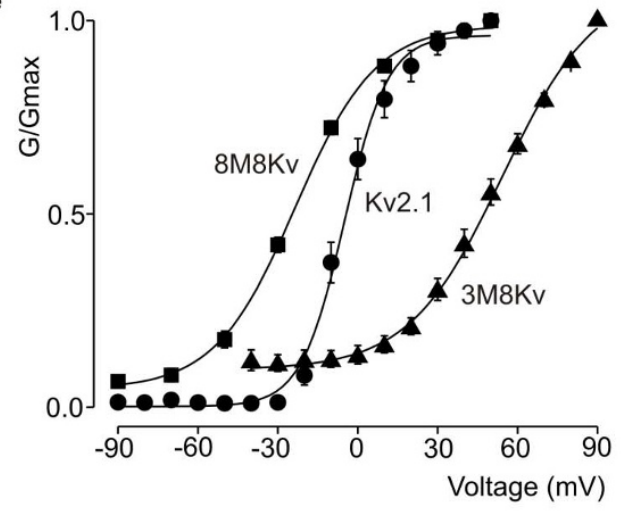

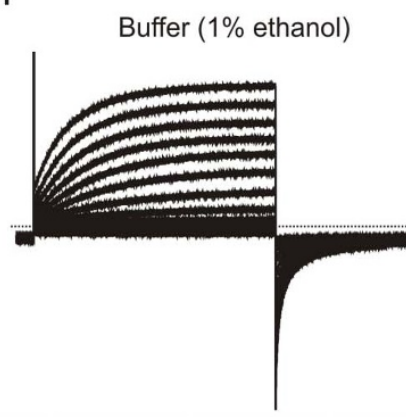

$3 \mathrm{M} 8 \mathrm{Kv}$

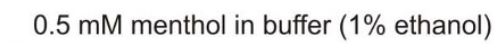

Figure 4 | Chimeras of S3-S4 region of TRPM8 transplanted into Kv2.1. (a) Alignment of TRPM8 and Kv2.1, and design of chimeras. (b) Families of current traces for Kv2.1 after subtraction of capacitive and leak currents using agitoxin2. Holding and tail voltages were $-70 \mathrm{mV}$, and depolarizations were from $-90 \mathrm{mV}$ to $+70 \mathrm{mV}$ in $10 \mathrm{mV}$ increments. (c) Families of current traces for $8 \mathrm{M} 8 \mathrm{Kv}$ after subtraction of capacitive and leak currents using agitoxin2. Holding and tail voltages were $-100 \mathrm{mV}$, and depolarizations were from $-90 \mathrm{mV}$ to $+50 \mathrm{mV}$ in $20 \mathrm{mV}$ increments. (d) Families of unsubtracted current traces for $3 \mathrm{M} 8 \mathrm{Kv}$ without subtraction of capacitive and leak currents. Holding and tail voltage was $-70 \mathrm{mV}$, and depolarizations were from $-40 \mathrm{mV}$ to $+90 \mathrm{mV}$ in $10 \mathrm{mV}$ increments. (e) G-V plots of Kv2.1 and the chimeras $3 \mathrm{M} 8 \mathrm{Kv}$ and $8 \mathrm{M} 8 \mathrm{Kv}$. (f) Unsubtracted current traces for $3 \mathrm{M} 8 \mathrm{Kv}$ before and after treatment with menthol. Voltage protocol was identical to the one used in d. Error bars indicate s.e.m. $(\mathrm{n}=3)$. Dotted lines in current races represent zero current.

To explore this idea, we replaced portions of the S4 helix of TRPM8 (which has 2 Arg and 1 His) with those of the S4 helix of Kv2.1 (which has 6 positively charged residues). The chimera replacing the largest portion of the S4 helix in TRPM8 added one Arg residue, one Lys residue, and replaced a His with an Arg residue. However, all chimeras involving replacement of S3-S4 regions of TRPM8 with segments of the Kv2.1 paddle failed to give rise to either voltage- or menthol-activated currents (chimeras 1-4KvM8; Fig. 3).

S3-S4 chimeras between TRPV1 and Kv2.1. The sequence similarity between TRPV1 and Kv2.1 in the S3-S4 region is lower than that between TRPM8 and Kv2.1 (22\% compared to 31\%). Consequently, there are several alignments with similar homology that can be constructed between Kv2.1 and TRPV1. We therefore explored two alignments in the S3-S4 region to design chimeras between TRPV1 and Kv2.1 (Fig. 3a,b; 5a). In the first, the loop between the S3 and S4 helices of TRPV1 is longer than in the alternate alignment. Chimeras generated using both alignments gave rise to functional channels $(2 \mathrm{~V} 1 \mathrm{Kv}, 5 \mathrm{~V} 1 \mathrm{Kv}$, and $6 \mathrm{~V} 1 \mathrm{Kv})$, with the exception of $1 \mathrm{~V} 1 \mathrm{Kv}$. In contrast, when the same portion of the Kv2.1 paddle was replaced by the S3-S4 region of TRPV1 using the alternate alignment to generate chimera $5 \mathrm{~V} 1 \mathrm{Kv}$, a functional $\mathrm{Kv}$ 
Table 1 Voltage-activation relationships for Kv2.1 and chimeras with TRPVI and TRPM8

\begin{tabular}{lcr} 
Channel & $z$ & \multicolumn{1}{c}{$\mathrm{V}_{1 / 2}(\mathrm{mV})$} \\
\hline Kv2.1 & $3.2 \pm 0.3$ & $-4.9 \pm 0.9$ \\
$3 \mathrm{M} 8 \mathrm{Kv}$ & $1.5 \pm 0.1$ & $54.2 \pm 1.5$ \\
$8 \mathrm{M} 8 \mathrm{Kv}$ & $1.7 \pm 0.1$ & $-23.4 \pm 1.1$ \\
$2 \mathrm{~V} 1 \mathrm{Kv}$ & $0.9 \pm 0.1$ & $79.9 \pm 0.7$ \\
$5 \mathrm{~V} 1 \mathrm{Kv}$ & $1.3 \pm 0.1$ & $70.3 \pm 0.9$ \\
\hline
\end{tabular}

A single Boltzmann function was fit to $G-V$ relations to obtain $z$ and $V_{1 / 2}$ values. $n=3$ in all cases.

channel was obtained (Fig. $5 \mathrm{~d}$ and e), suggesting that the transferred region in this case was more compatible with the structure of Kv2.1 than for $1 \mathrm{~V} 1 \mathrm{Kv}$. Similar to what was observed for the chimeras between Kv2.1 and TRPM8 discussed above, all functional chimeras were sensitive to agitoxin2, and had a reversal potential of $\sim-20 \mathrm{mV}$.

Several of the functional paddle chimeras exhibited constitutive activity and could not be fully closed with membrane hyperpolarization even though they retained some voltage-sensitivity (for example, $2 \mathrm{~V} 1 \mathrm{Kv}$ and $6 \mathrm{~V} 1 \mathrm{Kv}$; Fig. $5 \mathrm{~b}, \mathrm{c}$, and e), resembling the $3 \mathrm{M} 8 \mathrm{Kv}$ chimera discussed earlier. All these chimeras exhibit altered G-V relations, with slopes much lower than observed for Kv2.1 (Fig. 5e; Table 1). In the case of the $6 \mathrm{~V} 1 \mathrm{Kv}$ chimera, the $\mathrm{G}-\mathrm{V}$ relation is so shallow and complex that it cannot be well-defined with a single Boltzmann function (Fig. 5e).

Residues in the S3-S4 region of TRPV1 shown in green in Fig. 5a have been demonstrated to be important for activation of the channel by capsaicin and $\mathrm{RTX}^{9,10}$. If these residues contribute to forming the receptor for these ligands, transferring the S3-S4 region of TRPV1 into Kv2.1 might render the chimeras sensitive to capsaicin. However, the largest of these chimeras $(5 \mathrm{~V} 1 \mathrm{Kv})$ was not sensitive to high concentrations of capsaicin even though it gave rise to robust voltage-activated currents (Fig. 5f). Similar to what we observed with TRPM8 chimeras, all reverse chimeras where portions of the S3-S4 region of TRPV1 were replaced by those of the Kv2.1 paddle failed to give rise to either voltage- or capsaicin-activated currents $(1-4 \mathrm{KvV} 1$, Fig. 3).

Previous studies suggest that capsaicin binds to the internal regions between the $\mathrm{S} 2$ and $\mathrm{S} 3$ helices of $\mathrm{TRPV} 1^{8}$. If this idea is correct, the lack of capsaicin sensitivity of the S3-S4 chimeras $2 \mathrm{~V} 1 \mathrm{~K}, 5 \mathrm{~V} 1 \mathrm{Kv}$, and $6 \mathrm{~V} 1 \mathrm{Kv}$ is not surprising as they do not contain any portion of the S2-S3 linker region of TRPV1. In an effort to render the $\mathrm{Kv}$ channel sensitive to capsaicin, we swapped the internal regions of the S2 and S3 helices in Kv2.1 with those of TRPV1 to generate the $8 \mathrm{~V} 1 \mathrm{Kv}$ chimera (Fig. 3 ). This chimera did not give rise to either voltage- or capsaicin-activated currents, suggesting that it is non-functional.

S1-S4 chimeras. The S1-S4 domain of $\mathrm{Kv}$ channels can be transferred to channels that are not voltage-activated, endowing them with voltage-sensitivity ${ }^{41,42}$, demonstrating that the voltage sensor is an independent modular domain. Moreover, other voltage sensing proteins have been discovered that contain an S1$\mathrm{S} 4$ domain without a separate pore domain, such as $\mathrm{Ci}-\mathrm{VSP}^{47}$, and $\mathrm{Hv}^{48,49}$. Taken together, these observations suggest that nature utilizes the S1-S4 domain as a general scaffold to sense voltage. To test whether the S1-S4 regions of TRP channels have similar modular characteristics, we swapped the S1-S4 of TRPV1 and TRPM8 with that of Kv2.1, and also generated the reverse chimeras. All these chimeras $(13 \mathrm{M} 8 \mathrm{Kv}, 14 \mathrm{M} 8 \mathrm{Kv}, 9 \mathrm{~V} 1 \mathrm{Kv}, 10 \mathrm{~V} 1 \mathrm{Kv}, 5 \mathrm{KvM} 8,6 \mathrm{KvM} 8$, $5 \mathrm{KvV} 1$ and $6 \mathrm{KvV} 1$ depicted in Fig. 3) did not give rise to voltageor ligand-activated currents and were judged to be non-functional. We reasoned that these chimeras may have disrupted critical interactions between the S1 helix and pore helices of Kv2.150, resulting in a loss of channel function. To address this possibility, we created several S2-S4 chimeras, all of which were also nonfunctional (chimeras $15 \mathrm{M} 8 \mathrm{Kv}, 11 \mathrm{~V} 1 \mathrm{Kv}, 7 \mathrm{KvM} 8$, and $7 \mathrm{KvV} 1$ ).

S5-S6 pore chimeras. In addition to serving as the ion permeation pathway, the pore region of TRP channels plays critical roles in channel gating and pharmacology. For example, the outer pore domains of TRPV $1^{13}$ and TRPV $3^{51}$ have been implicated in temperature sensing, and DkTx and the vanillotoxins are believed to activate TRPV1 by binding to its pore region ${ }^{11,22,23}$. Motivated by the putative functional importance of the pore domain in TRP channel function, we replaced the S5-S6 pore region of Kv2.1 with the pore regions of TRPV1 and TRPM8 (chimeras $16-18 \mathrm{M} 8 \mathrm{Kv}$ and $12-14 \mathrm{~V} 1 \mathrm{Kv}$; Fig. 3). We also generated the reverse chimeras (8$9 \mathrm{KvM} 8$ and $8-9 \mathrm{KvV} 1$; Fig. 3) where the pore regions of TRPV1 and TRPM8 were replaced by the Kv2.1 pore domain. However, none of these chimeras gave rise to voltage-activated currents, even for $17 \mathrm{M} 8 \mathrm{Kv}, 18 \mathrm{M} 8 \mathrm{Kv}, 13 \mathrm{~V} 1 \mathrm{Kv}$ and $14 \mathrm{~V} 1 \mathrm{Kv}$, where the boundaries of the transferred region should not disrupt critical interactions between the S4-S5 linker and S6 helix defined for Kv channels ${ }^{41,42}$. We also investigated the DkTx-sensitivity of the three chimeras where the pore domain of Kv2.1 was replaced by that of TRPV1 $(12-14 \mathrm{~V} 1 \mathrm{Kv})$, but in each instance we could not observe measurable currents in response to application of $1 \mu \mathrm{M}$ DkTx to the external recording solution, even when testing over a wide range of membrane voltages. Because heterologous expression of TRPV1 is more efficient in mammalian cells compared to oocytes, we transfected HEK-293 cells with a few of the chimeras $(4 \mathrm{KvV} 1$, $5 \mathrm{KvV} 1,8 \mathrm{KvV} 1$, and $12 \mathrm{~V} 1 \mathrm{Kv}$ ) and used whole-cell patch clamp recordings to look for evidence of functional channels. In these experiments, none of the chimeras gave rise to voltage-, capsaicinor DkTx-activated currents that were distinguishable from nontransfected cells, confirming that they are non-functional.

\section{Discussion}

The primary objective of the present study was to establish structural relationships between TRP channels, for which little structural information is available, and $\mathrm{Kv}$ channels, for which a variety of Xray structures have been solved ${ }^{20,28,43}$. One of the interesting findings in the present study is that transfer of S3-S4 regions of TRPV1 and TRPM8 into the paddle motif of Kv2.1 resulted in functional voltageactivated channels (Fig. 3, 4, and 5), even though this region of Kv2.1 and the two TRP channels has low sequence homology. When compared to the other "paddle chimeras" that have been generated and tested $^{32,34}$, our constructs possess the lowest sequence homology between the two proteins within the transferred region. Indeed, TRPM8 and Kv2.1 have a sequence similarity of $31 \%$ in the paddle region and TRPV1 and Kv2.1 have a sequence similarity of $22 \%$, as compared to $40-45 \%$ between KvAP, Hv1, Nav2.1, Ci-VSP and $\mathrm{Kv} 2.1$. Our results strengthen the notion that $\mathrm{Kv}$ channel paddles lie in a remarkably unconstrained environment and probably make few critical contacts with the rest of the protein ${ }^{19,20,28,30,32,34,52}$.

One interesting feature of several of the present paddle chimeras is that they displayed constitutive activity at negative membrane voltages (Fig. 4, 5). Constitutive activity has been observed in Kv channels with mutations in the S4-S5 linker or S6 helices, implicating those regions in coupling voltage-sensor movement and opening/

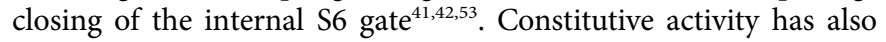
been observed in the Shaker Kv channel, where multiple S4 arginine residues of the channel were neutralized and a voltage-independent conductance was observed ${ }^{54}$. In that case constitutive activity was only observed when three of the outer four arginine residues were neutralized at the R1, R2 and R4 positions, whereas our constitutively activated chimeras involved neutralization of R2 or R2 and R3. Kv2.1 already contains a glutamine at the $\mathrm{R} 1$ position, but has two additional arginine residues $\mathrm{N}$-terminal to the canonical S4 arginine 
TRPV1 (507) SYSEILFFVOSLFMTVSVVLYFSORKEYVASMVFSLAMGWTNMLYYTRGEOOMGIYAV KV2 . 1 (253) GPLNAIDLLAILPYYVTIFLTESNKSVLQFQNVR----RVVQIFRIMRILRILKLARH

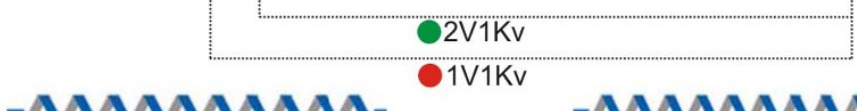

MMMMM-

WMWMW-

TRPV1 ( 507 ) SYSEILFFVQSLFMLVSVVLYF SQRKEYVASM--VFSLAMGWTNMLYYTRGFQQ

KV2. 1 (253) GPLNAIDLLAILPYYVTIFLTESNKSVLQFQNVRRVVQIFRIMRILRILKLARH

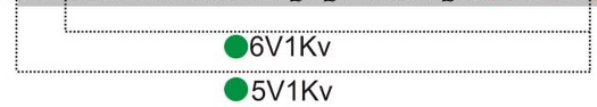

b

$2 \mathrm{~V} 1 \mathrm{Kv}$

C

$6 \mathrm{~V} 1 \mathrm{Kv}$
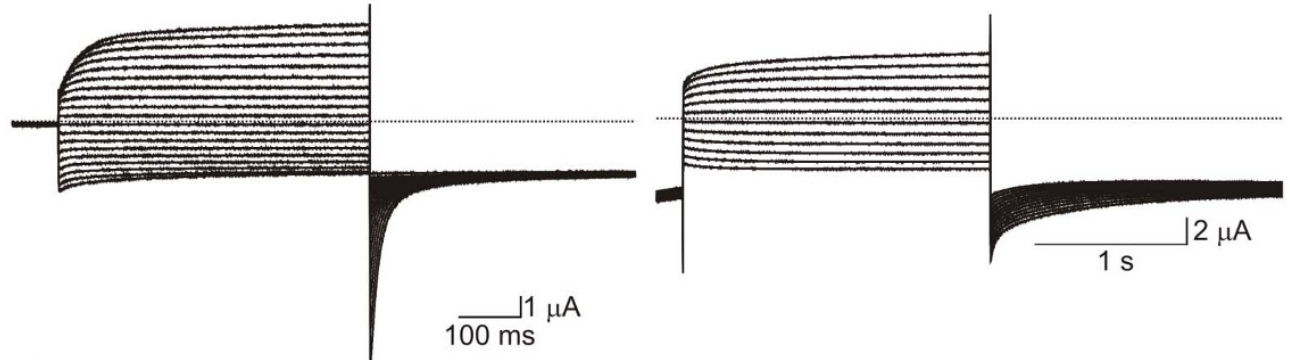

d

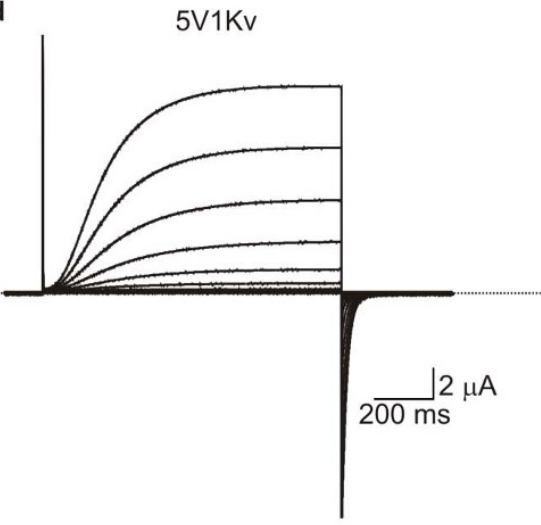

e

f
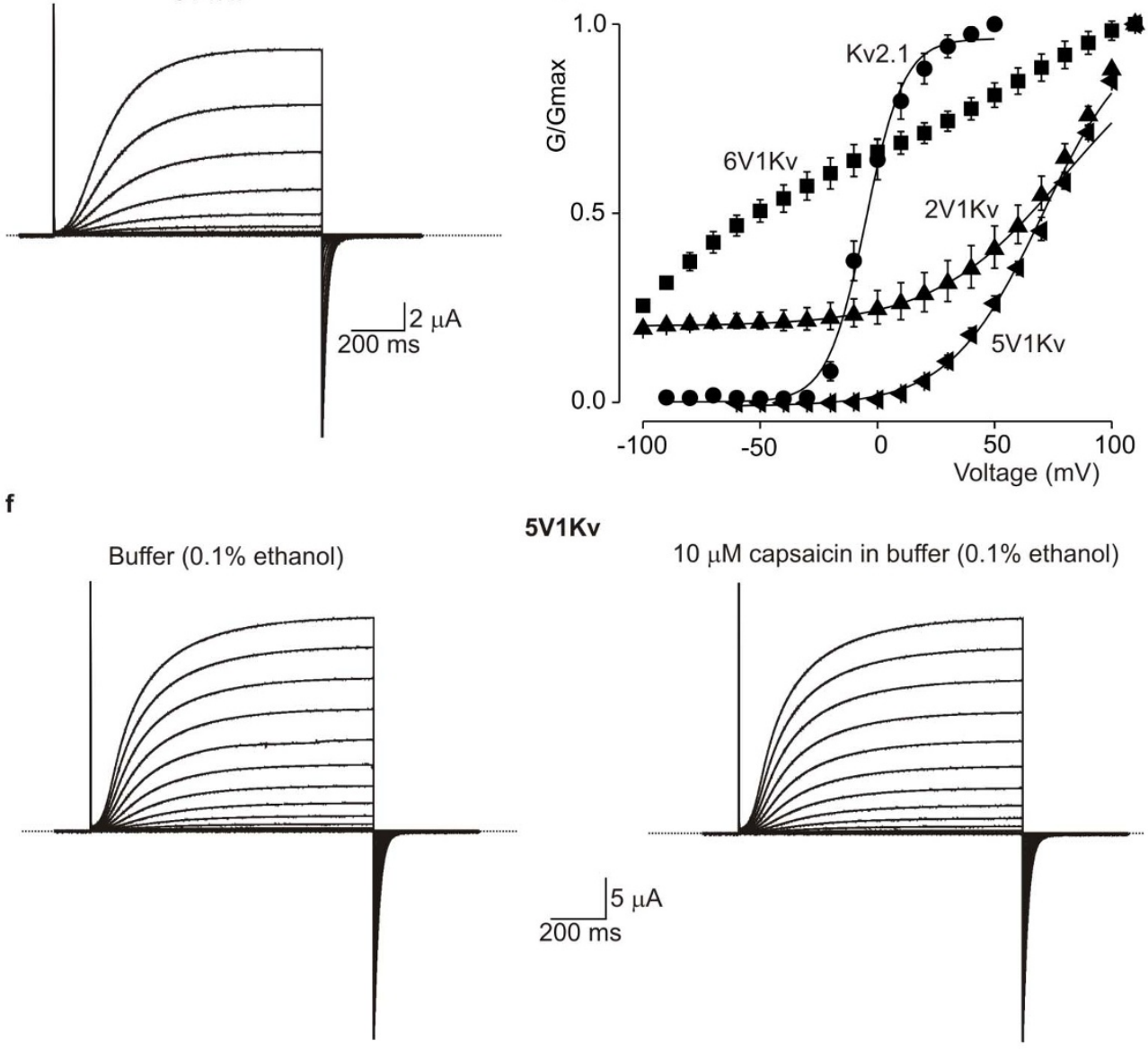

$5 \mathrm{~V} 1 \mathrm{Kv}$

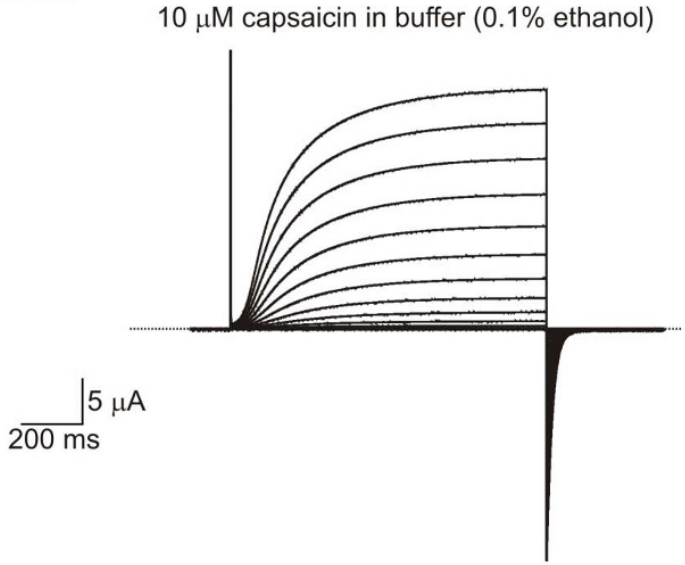

Figure $5 \mid$ Chimeras of S3-S4 region of TRPV1 transplanted into Kv2.1. (a) Alignments of TRPV1 and Kv2.1 and design of chimeras. (b) Families of current traces for $2 \mathrm{~V} 1 \mathrm{Kv}$ after subtraction of capacitive and leak currents using agitoxin2. Holding voltage was $-20 \mathrm{mV}$, tail voltage was $-100 \mathrm{mV}$ and depolarizations were from $-100 \mathrm{mV}$ to $+80 \mathrm{mV}$ in $10 \mathrm{mV}$ increments. (c) Families of current traces for 6V1Kv after subtraction of capacitive and leak currents using agitoxin2. Holding and tail voltage was $-100 \mathrm{mV}$, and depolarizations were from $-80 \mathrm{mV}$ to $+30 \mathrm{mV}$ in $10 \mathrm{mV}$ increments.

(d) Unsubtracted families of current traces for $5 \mathrm{~V} 1 \mathrm{Kv}$. Holding and tail voltage was $-80 \mathrm{mV}$, and depolarizations were from $-60 \mathrm{mV}$ to $+60 \mathrm{mV}$ in $10 \mathrm{mV}$ increments. (e) G-V plots of Kv2.1 and the chimeras. (f) Unsubtracted current traces for 5V1Kv before and after treatment with capsaicin. The voltage protocol was identical to the one used in d. Error bars indicate s.e.m. $(\mathrm{n}=3)$. Dotted lines in current races represent zero current. 
residues, both of which are neutralized in our constitutively active chimeras. Our chimeras also contain many other mutations in the transferred region, making it difficult to ascribe the constitutively activity to arginine neutralizations per se. Nevertheless, these collective results demonstrate that mutations in the voltage sensors, in addition to the S4-S5 linker and S6 gate can influence the coupling mechanism.

Although a strong indirect case can be made for structural similarities between TRP channels and Kv channels, as reviewed in the introduction, the vast majority of the chimeras we generated did not form functional channels. This outcome may help to explain why a majority of the functional chimeras involving TRP channels have been generated between orthologs of the same subtype. For example, chimeras between the rat and the avian orthologs of TRPV1 provided critical insights into the molecular determinants of vanilloid binding to the channel ${ }^{8}$, and chimeras between rat and Xenopus TRPV1 provided evidence implicating the pore region of the channel as the binding site of $\mathrm{DkTx}^{11}$. Similarly, chimeras created between rat and chicken TRPM8 led to the identification of specific residues of TRPM8 that are involved in icilin sensitivity ${ }^{17}$. In contrast to these examples of functional chimeras between TRP channel orthologs, there are few reports on chimeras between TRP channels belonging to different subtypes. The prominent outliers include reports on TRPV1-TRPV2 chimeras $^{14,55}$ and those between TRPV1 and TRPM $8^{56}$. Collectively, these results lead us to believe that the transmembrane regions of TRP channels have more constraining packing interactions than have been observed in X-ray structures of Kv channels ${ }^{20,28,43}$, where S1-S4 domains are loosely associated with the central pore domain and the paddle motif is relatively unconstrained (Fig. 1b). We speculate the structures of transmembrane regions of TRP channels are more closely related to that observed in the X-ray structure of $\mathrm{MlotiK}^{45}$, a prokaryotic tetrameric $\mathrm{K}$ channel containing a cytoplasmic cyclic nucleotide-binding domain, in which the helices within the S1-S4 domains exhibit extensive and tight packing interactions with each other and with the S5-S6 helices forming the central pore domain.

\section{Methods}

Channel constructs and chimera design. The Kv2.1 $\Delta 7$ channel was used because this Kv2.1 construct is sensitive to agitoxin $2^{57}$, enabling the toxin to be used to identify currents associated with chimeras containing the pore region of this channel. The rat orthologs of TRPV $1^{58}$ and TRPM $8^{59}$ were utilized for all experiments, and were a generous gift from David Julius (UCSF). Chimeras were generated by utilizing an overlap PCR approach.

Chimeras between Kv2.1 and TRPV1/TRPM8 were designed based on the sequence alignments shown in Fig. 2 and 3. These alignments were generated by initially using the AlignX tool of the Vector NTI software (Invitrogen) to align the sequences of tetrameric six transmembrane cation channels and to S1-S4 containing proteins, including TRPV1, TRPM8, Kv2.1, Kv1.2, Shaker, KvAP, Ci-VSP, Hv1, rNav1.2, NavAb and MlotiK. The alignment thus generated was further adjusted manually to improve homology with transmembrane helices, in particular for residues known to be structurally and functionally critical in $\mathrm{Kv}$ channels. The start and end points of transmembrane regions for Kv1.2, KvAP, NavAb, and MlotiK were obtained by visualizing their respective high resolution crystal structures, and those for other channels shown in Fig. 2 were predicted based on their sequence alignments with these four proteins

Electrophysiology. DkTx was produced recombinantly and agitoxin2 was synthesized by solid-phase methods as described previously ${ }^{22}$. Oocytes for chimera expression were obtained as previously described ${ }^{53}$.

Two-electrode voltage-clamp recordings were performed using an OC-725C oocyte clamp amplifier (Warner Instruments). Data was filtered at $1 \mathrm{kHz}$ (8 pole Bessel), and digitized at $10 \mathrm{kHz}$. Microelectrode resistances were between 0.1-1.2 M $\Omega$ when filled with $3 \mathrm{M} \mathrm{KCl}$. Solutions for recording chimeras with the Kv channel pore contained (in $\mathrm{mM}$ ) $\mathrm{KCl}(50), \mathrm{NaCl}(50), \mathrm{MgCl}_{2}$ (1), $\mathrm{CaCl}_{2}(0.3)$, and $\operatorname{HEPES}(20)$, at $\mathrm{pH} 7.4$ (pH adjusted with $\mathrm{NaOH}$ ). For recording currents from chimeras that contained TRP channel pores, $\mathrm{CaCl}_{2}$ was replaced with $\mathrm{BaCl}_{2}$. Unless otherwise stated, capacitive and background currents were identified by first blocking the Kv channel with agitoxin2, and then subtracting them to generate the currents shown in Fig. 4 and 5 .

HEK-293 cells for whole-cell patch clamp recordings were split on glass coverslips in a six-well plate, transfected with $1 \mu \mathrm{g}$ DNA per well and used 12-48 h after transfection. Whole-cell currents were recorded using an Axopatch $200 \mathrm{~B}$ patch clamp amplifier (Axon Instruments), filtered at $10 \mathrm{kHz}$ (8 pole Bessel), and digitized at $50 \mathrm{kHz}$. Microelectrode resistances were between 1-4 M $\Omega$ when filled with pipette solutions. External solution for recording currents from the chimera with the $\mathrm{Kv}$ channel pore (8KvV1) contained (in $\mathrm{mM}) \mathrm{KCl}(45), \mathrm{NaCl}(100), \mathrm{MgCl}_{2}$ (0.5), $\mathrm{CaCl}_{2}$ (2), and HEPES (10) at $\mathrm{pH} 7.2$ (pH adjusted with $\mathrm{NaOH}$ ), whereas the pipette solution contained (in mM) KCl (160), EGTA (1), $\mathrm{MgCl}_{2}(0.5)$, and HEPES (10) at pH 7.4 ( $\mathrm{pH}$ adjusted with $\mathrm{NaOH}$ ). External solution for recording the chimeras with TRP channel pores $(4 \mathrm{KvV} 1,5 \mathrm{KvV} 1$, and $12 \mathrm{~V} 1 \mathrm{Kv}$ ) contained (in $\mathrm{mM}) \mathrm{KCl}(2.8), \mathrm{NaCl}$ (150), $\mathrm{MgSO}_{4}(1)$, and HEPES (10) at $\mathrm{pH} 7.4(\mathrm{pH}$ adjusted with $\mathrm{NaOH})$, whereas the pipette solution contained (in $\mathrm{mM}$ ) $\mathrm{CsMeSO}_{3}$ (130), $\mathrm{CsCl}$ (15), $\mathrm{NaCl}$ (4), EGTA (5), and HEPES (10), at $\mathrm{pH} 7.4$ ( $\mathrm{pH}$ adjusted with $\mathrm{CsOH}$ ).

Conductance $(\mathrm{G})$-Voltage $(\mathrm{V})$ relationships were obtained by measuring tail currents following depolarization to test voltages as indicated in Fig. 4 and 5. A single Boltzmann function was fitted to the data according to the equation,

$\mathrm{G} / \mathrm{G}_{\max }=\left[1+\exp \left(-z \mathrm{~F}\left(\mathrm{~V}-\mathrm{V}_{1 / 2}\right) / \mathrm{RT}\right)\right]^{-1}$.

1. Montell, C. \& Rubin, G. M. Molecular characterization of the Drosophila trp locus: a putative integral membrane protein required for phototransduction. Neuron 2, 1313-1323 (1989).

2. Gaudet, R. Divide and conquer: high resolution structural information on TRP channel fragments. J Gen Physiol 133, 231-237 (2009).

3. Baez-Nieto, D., Castillo, J. P., Dragicevic, C., Alvarez, O. \& Latorre, R. Thermo TRP channels: biophysics of polymodal receptors. Advances in experimental medicine and biology 704, 469-490 (2011).

4. Bohlen, C. J. \& Julius, D. Receptor-targeting mechanisms of pain-causing toxins: How ow? Toxicon 60, 254-264 (2012).

5. Ramsey, I. S., Delling, M. \& Clapham, D. E. An introduction to TRP channels. Annu Rev Physiol 68, 619-647 (2006).

6. Voets, T., Talavera, K., Owsianik, G. \& Nilius, B. Sensing with TRP channels Nature chemical biology 1, 85-92 (2005).

7. DeFalco, J., Duncton, M. A. \& Emerling, D. TRPM8 biology and medicinal chemistry. Current topics in medicinal chemistry 11, 2237-2252 (2011).

8. Jordt, S. E. \& Julius, D. Molecular basis for species-specific sensitivity to "hot" chili peppers. Cell 108, 421-430 (2002).

9. Gavva, N. R. et al. Molecular determinants of vanilloid sensitivity in TRPV1. J Biol Chem 279, 20283-20295 (2004).

10. Chou, M. Z., Mtui, T., Gao, Y. D., Kohler, M. \& Middleton, R. E. Resiniferatoxin binds to the capsaicin receptor (TRPV1) near the extracellular side of the S4 transmembrane domain. Biochemistry 43, 2501-2511 (2004).

11. Bohlen, C. J. et al. A bivalent tarantula toxin activates the capsaicin receptor, TRPV1, by targeting the outer pore domain. Cell 141, 834-845 (2010).

12. Cui, Y. et al. Selective disruption of high sensitivity heat activation but not capsaicin activation of TRPV1 channels by pore turret mutations. J Gen Physiol 139, 273-283 (2012)

13. Grandl, J. et al. Temperature-induced opening of TRPV1 ion channel is stabilized by the pore domain. Nature neuroscience 13, 708-714 (2010).

14. Yao, J., Liu, B. \& Qin, F. Modular thermal sensors in temperature-gated transient receptor potential (TRP) channels. Proceedings of the National Academy of Sciences of the United States of America 108, 11109-11114 (2011).

15. Liu, B., Yao, J., Wang, Y., Li, H. \& Qin, F. Proton inhibition of unitary currents of vanilloid receptors. J Gen Physiol 134, 243-258 (2009).

16. Voets, T., Owsianik, G., Janssens, A., Talavera, K. \& Nilius, B. TRPM8 voltage sensor mutants reveal a mechanism for integrating thermal and chemical stimuli. Nature chemical biology 3, 174-182 (2007).

17. Chuang, H. H., Neuhausser, W. M. \& Julius, D. The super-cooling agent icilin reveals a mechanism of coincidence detection by a temperature-sensitive TRP channel. Neuron 43, 859-869 (2004).

18. Bandell, M. et al. High-throughput random mutagenesis screen reveals TRPM8 residues specifically required for activation by menthol. Nature neuroscience 9 , 493-500 (2006)

19. Swartz, K. J. Sensing voltage across lipid membranes. Nature 456, 891-897 (2008).

20. Long, S. B., Tao, X., Campbell, E. B. \& MacKinnon, R. Atomic structure of a voltage-dependent $\mathrm{K}+$ channel in a lipid membrane-like environment. Nature 450, 376-382 (2007).

21. Swartz, K. J. Tarantula toxins interacting with voltage sensors in potassium channels. Toxicon 49, 213-230 (2007).

22. Bae, C. et al. High Yield Production and Refolding of the Double-Knot Toxin, an Activator of TRPV1 Channels. PloS one 7 (12): e51516. doi:10.1371/ journal.pone.0051516 (2012).

23. Siemens, J. et al. Spider toxins activate the capsaicin receptor to produce inflammatory pain. Nature 444, 208-212 (2006).

24. Hill, K. \& Schaefer, M. TRPA1 is differentially modulated by the amphipathic molecules trinitrophenol and chlorpromazine. J Biol Chem 282, 7145-7153 (2007).

25. Armstrong, C. M. Interaction of tetraethylammonium ion derivatives with the potassium channels of giant axons. J Gen Physiol. 58, 413-437 (1971).

26. Jara-Oseguera, A., Llorente, I., Rosenbaum, T. \& Islas, L. D. Properties of the inner pore region of TRPV1 channels revealed by block with quaternary ammoniums. J Gen Physiol 132, 547-562 (2008). 
27. Holmgren, M., Smith, P. L. \& Yellen, G. Trapping of organic blockers by closing of voltage-dependent $\mathrm{K}+$ channels: evidence for a trap door mechanism of activation gating. J Gen Physiol 109, 527-535 (1997).

28. Jiang, Y. et al. X-ray structure of a voltage-dependent K+ channel. Nature $\mathbf{4 2 3}$, 33-41 (2003).

29. Jiang, Y., Ruta, V., Chen, J., Lee, A. \& MacKinnon, R. The principle of gating charge movement in a voltage-dependent K+ channel. Nature 423, 42-48 (2003).

30. Ruta, V., Chen, J. \& MacKinnon, R. Calibrated measurement of gating-charge arginine displacement in the KvAP voltage-dependent $\mathrm{K}+$ channel. Cell 123, 463-475 (2005).

31. Swartz, K. J. \& MacKinnon, R. Mapping the receptor site for hanatoxin, a gating modifier of voltage- dependent K+ channels. Neuron 18, 675-682 (1997b).

32. Alabi, A. A., Bahamonde, M. I., Jung, H. J., Kim, J. I. \& Swartz, K. J. Portability of paddle motif function and pharmacology in voltage sensors. Nature 450, 370-375 (2007).

33. Milescu, M. et al. Tarantula toxins interact with voltage sensors within lipid membranes. J Gen Physiol 130, 497-511 (2007).

34. Bosmans, F., Martin-Eauclaire, M. F. \& Swartz, K. J. Deconstructing voltage sensor function and pharmacology in sodium channels. Nature 456, 202-208 (2008).

35. Milescu, M. et al. Interactions between lipids and voltage sensor paddles detected with tarantula toxins. Nat Struct Mol Biol 16, 1080-1085 (2009).

36. Ahern, C. A. \& Horn, R. Specificity of charge-carrying residues in the voltage sensor of potassium channels. J Gen Physiol 123, 205-216 (2004).

37. Aggarwal, S. K. \& MacKinnon, R. Contribution of the S4 segment to gating charge in the Shaker K+ channel. Neuron 16, 1169-1177 (1996).

38. Seoh, S. A., Sigg, D., Papazian, D. M. \& Bezanilla, F. Voltage-sensing residues in the S2 and S4 segments of the Shaker K+ channel. Neuron 16, 1159-1167 (1996).

39. Salazar, H. et al. Structural determinants of gating in the TRPV1 channel. Nat Struct Mol Biol 16, 704-710 (2009).

40. Liu, Y., Holmgren, M., Jurman, M. E. \& Yellen, G. Gated access to the pore of a voltage-dependent K+ channel. Neuron 19, 175-184 (1997).

41. Lu, Z., Klem, A. M. \& Ramu, Y. Coupling between Voltage Sensors and Activation Gate in Voltage-gated $\mathrm{K}^{+}$Channels. J Gen Physiol 120, 663-676 (2002).

42. Lu, Z., Klem, A. M. \& Ramu, Y. Ion conduction pore is conserved among potassium channels. Nature 413, 809-813 (2001).

43. Long, S. B., Campbell, E. B. \& Mackinnon, R. Crystal structure of a mammalian voltage-dependent Shaker family K+ channel. Science 309, 897-903 (2005).

44. Payandeh, J., Scheuer, T., Zheng, N. \& Catterall, W. A. The crystal structure of a voltage-gated sodium channel. Nature 475, 353-358 (2011).

45. Clayton, G. M., Altieri, S., Heginbotham, L., Unger, V. M. \& Morais-Cabral, J. H. Structure of the transmembrane regions of a bacterial cyclic nucleotide-regulated channel. Proceedings of the National Academy of Sciences of the United States of America 105, 1511-1515 (2008).

46. Garcia, M. L., Garcia-Calvo, M., Hidalgo, P., Lee, A. \& MacKinnon, R. Purification and characterization of three inhibitors of voltage- dependent $\mathrm{K}+$ channels from Leiurus quinquestriatus var. hebraeus venom. Biochemistry 33, 6834-6839 (1994).

47. Murata, Y., Iwasaki, H., Sasaki, M., Inaba, K. \& Okamura, Y. Phosphoinositide phosphatase activity coupled to an intrinsic voltage sensor. Nature 435, 1239-1243 (2005).

48. Ramsey, I. S., Moran, M. M., Chong, J. A. \& Clapham, D. E. A voltage-gated proton-selective channel lacking the pore domain. Nature 440, 1213-1216 (2006).
49. Sasaki, M., Takagi, M. \& Okamura, Y. A Voltage Sensor-Domain Protein is a Voltage-Gated Proton Channel. Science 312, 589-592 (2006).

50. Lee, S. Y., Banerjee, A. \& MacKinnon, R. Two separate interfaces between the voltage sensor and pore are required for the function of voltage-dependent $\mathrm{K}(+)$ channels. PLoS biology 7, e47 (2009).

51. Grandl, J. et al. Pore region of TRPV3 ion channel is specifically required for heat activation. Nature neuroscience 11, 1007-1013 (2008).

52. Xu, Y., Ramu, Y. \& Lu, Z. A shaker $\mathrm{K}+$ channel with a miniature engineered voltage sensor. Cell 142, 580-589 (2010).

53. Sukhareva, M., Hackos, D. H. \& Swartz, K. J. Constitutive activation of the shaker kv channel. J Gen Physiol 122, 541-556 (2003).

54. Bao, H., Hakeem, A., Henteleff, M., Starkus, J. G. \& Rayner, M. D. Voltageinsensitive gating after charge-neutralizing mutations in the S4 segment of Shaker channels. J Gen Physiol 113, 139-151 (1999).

55. Jung, J. et al. Agonist recognition sites in the cytosolic tails of vanilloid receptor 1 . J Biol Chem 277, 44448-44454 (2002)

56. Brauchi, S., Orta, G., Salazar, M., Rosenmann, E. \& Latorre, R. A hot-sensing cold receptor: $\mathrm{C}$-terminal domain determines thermosensation in transient receptor potential channels. The Journal of neuroscience 26, 4835-4840 (2006).

57. Li-Smerin, Y. \& Swartz, K. J. Gating modifier toxins reveal a conserved structural motif in voltage- gated $\mathrm{Ca} 2+$ and $\mathrm{K}+$ channels. Proceedings of the National Academy of Sciences of the United States of America 95, 8585-8589 (1998).

58. Caterina, M. J. et al. The capsaicin receptor: a heat-activated ion channel in the pain pathway. Nature 389, 816-824 (1997).

59. McKemy, D. D., Neuhausser, W. M. \& Julius, D. Identification of a cold receptor reveals a general role for TRP channels in thermosensation. Nature 416, 52-58 (2002).

\section{Acknowledgments}

We thank Miguel Holmgren, Andres Jara-Oseguera, Dmitriy Krepkiy, Mark Mayer, Joe Mindell and members of the Swartz lab for helpful discussions. This work was supported by the Intramural Research Program of the National Institutes of Health National Institute of Neurological Disorders and Stroke (to K.J.S.), and by a National Institutes of Health National Institute of Neurological Disorders and Stroke competitive fellowship (to J.K.).

\section{Author contributions}

J. K. and K. J. S. designed the experiments, J. K. performed the experiments, and J. K. and K. J. S. wrote the paper.

\section{Additional information}

Competing financial interests: The authors declare no competing financial interests.

License: This work is licensed under a Creative Commons

Attribution-NonCommercial-ShareALike 3.0 Unported License. To view a copy of this license, visit http://creativecommons.org/licenses/by-nc-sa/3.0/

How to cite this article: Kalia, J. \& Swartz, K.J. Exploring structure-function relationships between TRP and Kv channels. Sci. Rep. 3, 1523; DOI:10.1038/srep01523 (2013). 